\title{
Erratum to: The treatment with pasireotide in Cushing's disease: effects of long-term treatment on tumor mass in the experience of a single center
}

\author{
Chiara Simeoli $^{1} \cdot$ Renata Simona Auriemma $^{2}$ Fabio Tortora ${ }^{3} \cdot$ Monica De Leo $^{1}$. \\ Davide Iacuaniello $^{1}$ - Alessia Cozzolino ${ }^{1}$ - Maria Cristina De Martino ${ }^{1}$. \\ Claudia Pivonello ${ }^{1} \cdot$ Ciro Gabriele Mainolfi $^{4} \cdot$ Riccardo Rossi $^{1} \cdot$ Sossio Cirillo $^{3}$. \\ Annamaria Colao ${ }^{1} \cdot$ Rosario Pivonello ${ }^{1}$
}

Published online: 8 April 2016

(C) Springer Science+Business Media New York 2016

\section{Erratum to: Endocrine (2015) 50:725-740}

DOI 10.1007/s12020-015-0557-2

In the original publication, some of the units in Tables 1,2 and 3 are not correctly reported; in detail, throughout the text and tables, pituitary tumor volume is expressed in $\mathrm{ml}$ instead of $\mathrm{mm}^{3}$. The corrected version of Tables 1,2 and 3 are given below.

The online version of the original article can be found under doi:10.1007/s12020-015-0557-2.

Rosario Pivonello

rosario.pivonello@unina.it

1 Dipartimento di Medicina Clinica e Chirurgia, Sezione di Endocrinologia, Università 'Federico II', Via Sergio Pansini 5, 80131 Naples, Italy

2 Ios \& Coleman Medicina Futura Medical Center, Naples, Italy

3 Department of Internal and Experimental Medicine, Division of Neurological Sciences, Second University of Naples, Naples, Italy

4 Dipartimento di Scienze Biomediche Avanzate, Università 'Federico II', Naples, Italy 
Table 1 Patient's profile at study entry

\begin{tabular}{ll}
\hline Patients number & 8 \\
Patients Age (years) & $38.9 \pm 17.6$ \\
Microadenoma nr. (\%) & $7(87.5)$ \\
Macroadenoma nr. $(\%)$ & $1(12.5)$ \\
Tumor volume $\left(\mathrm{mm}^{3}\right)$ & $416.46 \pm 937.07$ \\
UFC (nmol/24h) & $771.75 \pm 606.57$ \\
Median pasireotide starting dose $(\mu \mathrm{g} \mathrm{bid})$ & 900 \\
\hline
\end{tabular}

Table 2 Patient 1: Patient's profile before and after 3, 6, 12, 18, 21 and 24 months of pasireotide treatment

\begin{tabular}{|c|c|c|c|c|c|c|c|c|c|c|c|}
\hline Parameters & $\begin{array}{l}\text { Normal } \\
\text { range }\end{array}$ & Baseline & $\begin{array}{l}3 \\
\text { months }\end{array}$ & 6 months & $\Delta \%^{\mathrm{a}}$ & 12 months & $\Delta \%^{\mathrm{a}}$ & $\begin{array}{l}18 \\
\text { months }\end{array}$ & $\begin{array}{l}21 \\
\text { months }\end{array}$ & 24 months & $\Delta \%^{\mathrm{a}}$ \\
\hline Weight (kg) & - & 104.5 & 95.5 & 89 & -14.8 & 81.5 & -22 & 83 & 82.1 & 81 & -22.5 \\
\hline BMI $\left(\mathrm{kg} / \mathrm{m}^{2}\right)$ & $20-25$ & 35.3 & 32.2 & 30 & -15 & 27.5 & -22.1 & 28 & 27.7 & 27.3 & -22.7 \\
\hline $\begin{array}{l}\text { Waist circumference } \\
(\mathrm{cm})\end{array}$ & $<88$ & 119 & 112 & 103 & -13.4 & 104 & -12.6 & 98 & 103 & 97 & -18.5 \\
\hline Ferriman-Gallwey score & $<8$ & 30 & 32 & 28 & -6.7 & 23 & -23.3 & 19 & 18 & 14 & -53.3 \\
\hline HR QoL score & - & 41 & 48 & 56 & +36.6 & 50 & +21.9 & - & - & - & \\
\hline BDI-II score & - & 9 & 3 & 0 & -100 & 1 & -88.9 & 0 & 0 & 0 & -100 \\
\hline $\mathrm{SBP}(\mathrm{mmHg})$ & $<130$ & 120 & 115 & 120 & 0 & 125 & +4.2 & 130 & 120 & 112 & -6.7 \\
\hline DBP (mmHg) & $<85$ & 70 & 70 & 80 & +14.3 & 70 & 0 & 80 & 80 & 70 & 0 \\
\hline $\begin{array}{l}\text { Fasting glucose (mmol/ } \\
\text { L) }\end{array}$ & $3.9-6.9$ & 6.3 & 5.7 & 5.1 & -19 & 7.2 & +14.3 & 6.1 & 6.5 & 6.1 & -3.2 \\
\hline Insulin $(\mathrm{mU} / \mathrm{L})$ & $3-25$ & 81.6 & 21 & 83.3 & +2.1 & 14.8 & -81.9 & 24.4 & 14.6 & 40.3 & -50.6 \\
\hline HBA1C (\%) & $3.5-6$ & 5.5 & - & 6 & +9.1 & 6.9 & +25.4 & 6.6 & 6.9 & 7 & +27.3 \\
\hline $\begin{array}{l}\text { Total cholesterol } \\
(\mathrm{mmol} / \mathrm{L})\end{array}$ & $2.2-5.2$ & 5.3 & 4.8 & 4.9 & -7.5 & 4.8 & -9.4 & 4.8 & 5.1 & 4.8 & -9.4 \\
\hline $\begin{array}{l}\text { LDL cholesterol (mmol/ } \\
\text { L) }\end{array}$ & $1.6-3.3$ & 3.1 & 2.7 & 2.8 & -9.7 & 2.9 & -6.4 & 2.7 & 3 & 2.8 & -9.7 \\
\hline $\begin{array}{l}\text { HDL cholesterol (mmol/ } \\
\text { L) }\end{array}$ & $>1.3$ & 1.4 & 1.42 & 1.6 & +14.3 & 1.5 & +7.14 & 1.68 & 1.64 & 1.34 & -4.3 \\
\hline Triglycerides $(\mathrm{mmol} / \mathrm{L})$ & $0.3-1.7$ & 3.7 & 3.4 & 2.5 & -32.4 & 2.1 & -43.2 & 2.1 & 2.3 & 3.3 & -10.8 \\
\hline $\begin{array}{l}\text { BMD lumbar vertebrae } \\
\left(\mathrm{mg} / \mathrm{cm}^{3}\right)\end{array}$ & - & 0.962 & - & 1.011 & +5.1 & 1.002 & +4.1 & - & - & 0.983 & +2.2 \\
\hline $\begin{array}{l}\text { BMD proximal femur } \\
\text { total hip }\left(\mathrm{mg} / \mathrm{cm}^{3}\right)\end{array}$ & - & $\mathrm{NP}$ & - & 1.012 & - & NP & - & - & - & 0.916 & - \\
\hline $\begin{array}{l}\text { BMD proximal femur } \\
\text { neck }\left(\mathrm{mg} / \mathrm{cm}^{3}\right)\end{array}$ & - & NP & - & 0.972 & - & 0.838 & - & - & - & 0.856 & - \\
\hline Plasma ACTH (ng/L) & $0-46$ & 101 & 75 & 80 & -20.8 & 82 & -18.8 & 86 & 91 & 72 & -28.7 \\
\hline Serum cortisol $(\mathrm{nmol} / \mathrm{L})$ & $119-618$ & 634 & 440 & 628 & -0.95 & 574 & -9.5 & 498 & 682 & 381 & -39.9 \\
\hline Mean UFC (nmol/24 h) & $30-145$ & 535 & 132 & 138 & -74.2 & 162 & -69.7 & 127 & 145 & 111 & -79.2 \\
\hline $\begin{array}{l}\text { MRI: pituitary tumor } \\
\text { diameters }(\mathrm{mm})\end{array}$ & - & $15 \times 21 \times 17$ & - & $11 \times 15 \times 12$ & - & $10 \times 12 \times 8$ & - & - & - & $9 \times 12 \times 8$ & - \\
\hline $\begin{array}{l}\text { MRI: pituitary tumor } \\
\text { volume }\left(\mathrm{mm}^{3}\right)^{\mathrm{b}}\end{array}$ & - & 2731.05 & - & 1009.8 & -63 & 489.6 & -82 & - & - & 440.6 & -84 \\
\hline
\end{tabular}

BMI Body Mass Index, HR QoL Health-Related Quality of Life, BDI-II Beck Depression Inventory-II Questionnaire, SBP systolic blood pressure, $D B P$ diastolic blood pressure, $H b A 1 c$ glycated haemoglobin, $B M D$ bone mineral density, UFC urinary free cortisol, MRI magnetic resonance imaging, NP not performed

a vs baseline

b Tumor volume was calculated in line with the "Di Chiro \& Nelson" formula (volume $=$ height $\times$ length $\times$ width $\times$ n/6) 
Table 3 Patient 2: Patient's profile before and after 3, 6, 12, 18, 21 and 24 months of pasireotide treatment

\begin{tabular}{|c|c|c|c|c|c|c|c|c|c|c|c|}
\hline Parameters & $\begin{array}{l}\text { Normal } \\
\text { range }\end{array}$ & Baseline & $\begin{array}{l}3 \\
\text { months }\end{array}$ & 6 months & $\Delta \%^{\mathrm{a}}$ & $\begin{array}{l}12 \\
\text { months }\end{array}$ & $\Delta \%^{\mathrm{a}}$ & $\begin{array}{l}18 \\
\text { months }\end{array}$ & $\begin{array}{l}21 \\
\text { months }\end{array}$ & $\begin{array}{l}24 \\
\text { months }\end{array}$ & $\Delta \%^{\mathrm{a}}$ \\
\hline Weight (kg) & - & 61 & 60 & 56.5 & -7.4 & 52.5 & -13.9 & 55 & 56.7 & 56 & -8.2 \\
\hline BMI $\left(\mathrm{kg} / \mathrm{m}^{2}\right)$ & $20-25$ & 20.4 & 20 & 18.89 & -7.4 & 17.55 & -14 & 18.39 & 18.96 & 18.72 & -8.2 \\
\hline Waist circumference $(\mathrm{cm})$ & $<88$ & 80 & 80 & 84 & +5 & 72 & -10 & 75 & 75 & 73 & -8.75 \\
\hline Ferriman-Gallwey score & $<8$ & 11 & 3 & 1 & -90.9 & 1 & -91 & 1 & 1 & 1 & -90.9 \\
\hline HR QoL score & - & 37 & 31 & 35 & -5.4 & 41 & +10.8 & - & - & - & - \\
\hline BDI-II score & - & 21 & 23 & 20 & -4.8 & 17 & -19 & 12 & 8 & 6 & -71.4 \\
\hline $\mathrm{SBP}(\mathrm{mmHg})$ & $<130$ & 110 & 100 & 102 & -7.3 & 105 & -4.5 & 110 & 100 & 100 & -9.1 \\
\hline $\mathrm{DBP}(\mathrm{mmHg})$ & $<85$ & 70 & 70 & 60 & -14.3 & 65 & -7.1 & 70 & 70 & 60 & -14.3 \\
\hline Fasting glucose $(\mathrm{mmol} / \mathrm{L})$ & $3.9-6.9$ & 4.1 & 4.1 & 4.3 & +4.9 & 4.2 & +2.4 & 4.7 & 3.9 & 4.3 & +4.9 \\
\hline Insulin (mU/L) & $3-25$ & 21.3 & 7.7 & 5.9 & -72.3 & 5.4 & -74.6 & 4.8 & 16.1 & 5.4 & -74.6 \\
\hline HBA1C (\%) & $3.5-6$ & 5.4 & - & 5.6 & +3.7 & 5.3 & -1.85 & 5.6 & 5.7 & 5.8 & +7.4 \\
\hline $\begin{array}{l}\text { Total cholesterol (mmol/ } \\
\text { L) }\end{array}$ & $2.2-5.2$ & 5.4 & 4.8 & 4.2 & -22.2 & 4.5 & -16.7 & 5.1 & 5.6 & 5.4 & 0 \\
\hline $\begin{array}{l}\text { LDL cholesterol (mmol/ } \\
\text { L) }\end{array}$ & $1.6-3.3$ & 2.6 & 2.5 & 2.1 & -19.2 & 2.2 & -15.4 & 2.6 & 2.7 & 2.9 & +11.5 \\
\hline $\begin{array}{l}\text { HDL cholesterol (mmol/ } \\
\text { L) }\end{array}$ & $>1.3$ & 2.64 & 2.12 & 1.98 & -25 & 2.18 & -17.4 & 2.34 & 2.76 & 2.36 & -10.6 \\
\hline Triglycerides (mmol/L) & $0.3-1.7$ & 0.8 & 0.9 & 0.6 & -25 & 0.6 & -25 & 0.8 & 0.7 & 0.7 & -12.5 \\
\hline $\begin{array}{l}\text { BMD lumbar vertebrae } \\
\left(\mathrm{mg} / \mathrm{cm}^{3}\right)\end{array}$ & - & 0.822 & - & 0.815 & -0.6 & 0.8 & -2.4 & - & - & 0.769 & -6.2 \\
\hline $\begin{array}{l}\text { BMD proximal femur } \\
\text { total hip }\left(\mathrm{mg} / \mathrm{cm}^{3}\right)\end{array}$ & - & 0.656 & - & 0.647 & -0.5 & NP & - & - & - & 0.669 & +2.9 \\
\hline $\begin{array}{l}\text { BMD proximal femur } \\
\text { neck }\left(\mathrm{mg} / \mathrm{cm}^{3}\right)\end{array}$ & - & 0.723 & - & 0.726 & +0.4 & 0.473 & -34.6 & - & - & 0.748 & +3.45 \\
\hline Plasma ACTH (ng/L) & $0-46$ & 32 & 13 & 13 & -59.4 & 22 & -31.25 & 22 & 20 & 21 & -34.4 \\
\hline Serum cortisol (nmol/L) & $119-618$ & 495 & 287 & 322 & -34.9 & 512 & +3.4 & 584 & 492 & 573 & +15.7 \\
\hline Mean UFC (nmol/24 h) & $30-145$ & 580 & 20 & 48.5 & -91.6 & 85.75 & -85.2 & 88 & 182 & 221.5 & -61.8 \\
\hline $\begin{array}{l}\text { MRI: pituitary tumor } \\
\text { diameters }(\mathrm{mm})\end{array}$ & - & $6 \times 7 \times 6$ & - & $2.5 \times 2.8 \times 3$ & - & 0 & - & - & - & 0 & - \\
\hline $\begin{array}{l}\text { MRI: pituitary tumor } \\
\text { volume }\left(\mathrm{mm}^{3}\right)^{\mathrm{b}}\end{array}$ & - & 128.5 & - & 10.71 & -91.7 & 0 & -100 & - & - & 0 & -100 \\
\hline
\end{tabular}

$B M I$ Body Mass Index, HR QoL Health-Related Quality of Life, BDI-II Beck Depression Inventory-II Questionnaire, SBP systolic blood pressure, $D B P$ diastolic blood pressure, $H b A l c$ glycated haemoglobin, BMD bone mineral density, UFC urinary free cortisol, MRI magnetic resonance imaging, NP not performed

a vs baseline

b Tumor volume was calculated in line with the "Di Chiro \& Nelson" formula (volume $=$ height $\times$ length $\times$ width $\times$ п/6) 